\title{
ALK Positive
}

National Cancer Institute

\section{Source}

National Cancer Institute. ALK Positive. NCI Thesaurus. Code C128831.

Indicates that ALK expression has been detected in a sample. 